\title{
AN INTELLIGENT CHANNEL ESTIMATION METHOD FOR FUTURE MOBILE GENERATION
}

\author{
Khaled H. Almotairi \\ Department of Computer Engineering, College of Computers and Information Systems \\ Umm Al-Qura University, Saudi Arabia
}

\begin{abstract}
The challenges of the future generations of mobile telephony operators are based on the use of previous generation's connectivity. The signal reconstruction is a technique that requires in most of the cases, the insertion of Pilot Symbols (PS) to ensure identification data reception. The PS are sent through the network and are known by the receiver and the transmitter. Therefore, they occupy bandwidth but contain no information. For Channel Estimation (C.E.), the LTE-A standard adopts inserting a pilot symbol in seven data symbols for each subcarrier. This is reflected in a throughput loss of the transmitted information. This method considers that channel does not vary during the period of slot transmission, but the rapid time variation is one of channel characteristics. We propose in this work an intelligent solution for C.E. in order to reduce bit rate loss while ensuring a good quality. A combination of two techniques of Artificial Intelligence has been proposed: Deep Learning and Fuzzy Logic. The main motivation of such a combination is to incorporate the complementarity of these two techniques to exploit the advantage of each and overcome its limitation. The proposed method is characterized by a non-random initialization of his weights from a fuzzy rule base. The implementation of this smart system into a C.E. system has reduced the loss of throughput and follows the variations of the propagation channel. The proposed Hybrid Deep Learning model provides better results than classical estimators. It has, also, a low complexity and a fast treatment.
\end{abstract}

\section{KEYWORDS}

Channel estimation, interpolation, LTE-A, Deep Learning, Fuzzy Logic.

\section{INTRODUCTION}

Due to the evolution of mobile communications systems technologies, mobile users can benefit from wireless service with high data rates, secure and permanent connection. Developers and telecom operators had to offer new services with high bitrate. For three decades, new technologies in the mobile world have undergone a spectacular evolution. These technologies are characterized by several trends, the most significant is the explosion of the mobile internet and the growing number of connected devices. In this context, the emergence of new technologies meets the growing need to provide a new standard capable not only to offer the best flow but also to ensure adequate fluidity. High data rates and a secure, permanent connection have become an unavoidable necessity for all mobile users. For the fourth generation of cellular systems, it is through the Orthogonal Frequency Division Multiplexing: OFDM [1] that the highest rates in wireless systems are deployed. The Third Generation Partnership Project: 3GPP [2] is adopting the OFDM technique [3] to achieve high data rates. The Orthogonal Frequency Division Multiple Access (OFDMA) has been reserved for the downlink only. But for the uplink, 3GPP has 
International Journal of Wireless \& Mobile Networks (IJWMN) Vol. 12, No. 3, June 2020

adopted another technique which is a modified form of OFDMA, called "Single Carrier Frequency Multiple Access Division: SC-FDMA" [3] [4]. SC-FDMA is a single carrier access technique with similar throughput performance and essentially the same complexity. SC-FDMA has a significant advantage over OFDMA. It offers a low power peak ratio compared to the average noted: "PAPR: Peak to Average Power Ratio" [5] [6] [7]. The SC-FDMA is currently used as a multiple access technique for uplink for "Long Term Evolution: LTE" and for "Long Term Evolution Advanced: LTE-A".

The present work is organized in three axes. In the first part, we present mobile system and access technique evolution and the main characteristics of mobile radio channels, then a quick description of the family of OFDM systems by focusing on OFDMA techniques. The second part is devoted to the channel estimation using Least Square Estimator (LSE) and Minimum Mean Square Estimator (MMSE). The third part of this work deals with experiments and studies of complexity and convergence. We present the simulations made by the LSE and MMSE methods. We make a comparative study of the existing interpolation methods and present two models of classical neural networks as interpolators able to improve the performances of channel estimation system. Then, we focus our work on the contribution of deep neural networks for the detection of channel variations by using neuronal solution in order to improve the quality of interpolation. This technique aims to reduce the number of inserted pilot symbols, which can reduce throughput losses.

\section{Mobile Systems Evolution}

The universe has seen the birth of several generations of mobile telephony in a very short period. In the 80s, an evolution of analog technology to digital technology first, then in the 90s, changes in access techniques and especially rapid duplication of offered flows. And recently, this new century has enjoyed greedy services in broadband. The first mobile telephony standards (1G) had analog operation and consisted of relatively large devices. These were mainly standards called "Advanced Mobile Phone System: AMPS" [8]. The AMPS appeared in 1976 in the United States, was the first cellular network standard. It is an analog network that only had weak security mechanisms that made it possible to hack telephone lines and offered poor quality of service. The FDMA access technique is the basis of $1 \mathrm{G}$ systems. It is a greedy technique in terms of use of frequency band. This technique has been replaced by the technique "Time Division Multiple Access: TDMA". The TDMA uses a technique of temporal division of the communication channels in order to increase the volume of data transmitted simultaneously. After a short time, mobile communications systems have undergone other changes. The firstgeneration networks have been rendered obsolete with the appearance of a second generation entirely digital. The second generation of mobile networks $(2 \mathrm{G})$ marked a break with the first generation of cellular phones thanks to the shift from analogue to digital and the introduction of the concept of "roaming". The contribution of "roaming" is the fact of benefiting from the telephone services on his mobile, as well as the connection to the Internet from a foreign country following agreements between the various telephone operators. The main $2 \mathrm{G}$ mobile telephony standards are the "Global System for Mobile Communications: GSM"[9]. GSM was the most used standard in Europe at the end of the 20th century, supported in the United States. Two kinds of Mobile frequency bands are presented in Europe: $900 \mathrm{MHz}$ and $1800 \mathrm{MHz}$. In the United States, however, the frequency bands used are the $850 \mathrm{MHz}$ and $1900 \mathrm{MHz}$ bands. Thus, we call tri-band, mobile phones that can operate in Europe and the United States. $2 \mathrm{G}$ networks were a great evolution in the world of mobile networks, they made possible the transmission of digital data in addition to the voice. With the advent of $2 \mathrm{G}$, new services appeared, such as short text messages: "Short Message Service: SMS" and multimedia messages "MultiMedia Message Service MMS". With a bandwidth of up to $9.6 \mathrm{kbits} / \mathrm{s}$, the GSM standard has been able to 
provide a better quality of service than the $1 \mathrm{G}$ analog standard and has enabled services that are no longer content with voice. In order to open wide the door to multimedia applications, the GSM has experienced several extensions offering higher speeds. The system $2.5 \mathrm{G}$ and more, which allowed a greater flow accompanied by new services offered to users. $2.5 \mathrm{G}$ networks are networks that have replaced GSM and are improved versions of it. The "General Packet Radio System Standard: GPRS" provides speeds that can theoretically reach $114 \mathrm{kbits} / \mathrm{s}$. The "Enhanced Data rates for Global Evolution: EDGE" standard, another improved version of the second-generation network, has been named the $2.75 \mathrm{G}$ network. This solution has improved the throughput in a very important way that can theoretically reach $384 \mathrm{Kbits} / \mathrm{s}$. A little later, the mobile world saw the birth of the code access technique: The Code "Multiple Access Division: CDMA", using a spread spectrum technique to broadcast a radio signal over a wide range of frequencies. The use of the codes was used for spectral spreading and at the same time for the separation of user data thus allowing good spectral efficiency in comparison with TDMA.

The International Telecommunication Union (ITU) has introduced IMT-2000 specifications for International Mobile Telecommunications for the Year 2000 [10] that govern the characteristics of third generation $(3 \mathrm{G})$ mobile radio communications systems. $3 \mathrm{G}$ is a network with a very high transmission rate in comparison with older generations. The notion of universality has been introduced and especially the compatibility of the third-generation standard with second generation networks. The theoretical flow can reach $2 \mathrm{Mbits} / \mathrm{s}$ under very specific conditions. It was in 2002 that $3 \mathrm{G}$ marked the best evolution in the mobile world. The $3 \mathrm{G}$ standard offered a very high speed allowing a better quality for videoconferencing, the mobile Internet and the transmission of video at very high speed. This standard called "Universal Mobile Telecommunication System: UMTS" [9] is a broadband system up to $5 \mathrm{MHz}$. Access technology has evolved from CDMA to Wideband Code Multiple Access Division (WCDMA). The use of orthogonal codes of large lengths made it possible to multiply the transmission rate as a function of the length of the codes. It is from the third generation that we talk about the smart phone. The phone is transformed into a computer thanks to high data rates and internet access. $3.9 \mathrm{G}$ systems, also known as LTE, were born in the last few years, they operate in a band of $20 \mathrm{MHZ}$ and offer a bit rate of up to $100 \mathrm{Mbit} / \mathrm{s}$ in downlink and up to $50 \mathrm{Mbps}$ in uplink and offer bandwidth flexibility. LTE, like the $3 \mathrm{G}$ standard, uses packet switching but differs by the $3 \mathrm{G}$ standard because it combines frequency multiplexing and time division multiplexing that makes it able to significantly improve the flow rate by means of the Orthogonal Frequency Division Multiaccess: OFDM.

The frequency bands used by LTE varies between $1.4 \mathrm{MHz}$ and $20 \mathrm{MHz}$. The LTE-A is the most recent version of the LTE, it presents an evolution of the mobile network allowing to obtain theoretical speeds up to $300 \mathrm{Mb} / \mathrm{s}$. The stakes of the LTE-A for the mobile operators relies on the good use of the connectivity capabilities already offered by LTE and $3 \mathrm{G}$ networks, many messaging services, multimedia content exchange and even telephony have developed. The next generations of mobile radio standards are expanding and offer speeds of up to $1 \mathrm{~GB} / \mathrm{s}$. the new standards will theoretically exceed the bit rates offered by the optical fiber but in reality and because of the attenuations and weakening's, the actual bit rates can reach one hundred megabits per second. OFDM is a method of coding digital data over several carrier frequencies. A large number of close orthogonal subcarrier signals are used to carry data to multiple parallel data streams. Each subcarrier is modulated with a conventional modulation scheme at a low symbol rate, thus presenting the solution of access to the new generation. OFDMA and SC-FDMA are part of the OFDM family. The latter is the key to the success of current generations. 3GPP LTE has adopted the OFDMA for the uplink and the SC-FDMA for the downlink. OFDMA is the technique used for downlink in 4G mobile generation. SC-FDMA was chosen for the uplink mainly because of its low PAPR compared to the OFDMA coding. This choice made it possible 
to solve the amplification problems generated by the summation of the different signals emanating from different carriers. The low PAPR of the SC-FDMA is a result of the addition of a discrete Fourier transform with respect to the block of the OFDMA emission. This transformation of the time domain towards the frequency domain, also called recoding, weakens the amplitude variations. The SC-FDMA transmitter is only a modified OFDMA transmitter. The Fourier transform added to the transmitter will be compensated by the addition of an inverse Fourier transform on reception.

The rapid variation over time is one of the characteristics of a radio channel. Thus, the propagation medium has limitations due to distortions of the transmitted signal and causes interference problems at the reception. The most important mission of the receiver is to ensure a perfect reproduction of the transmitted signal, so we must know the different types of distortion made to the signal transmitted during its journey from the transmitter to the receiver. This identification of the distortion will allow it to perform the equalization operation well.

The reconstitution of the transmitted signal is a technique that requires in most cases the use of a training sequence to ensure the identification of the signals on reception. A training sequence is a set of data sent through the network and previously known by the receiver and the transmitter and therefore contain no information. The advantage of using learning sequences is knowing the characteristics of the channel, the disadvantage is the bandwidth occupation. The more frequent the use of training sequences, the more precise the knowledge of the characteristics of the channel. The 3GPP LTE standard adopts the insertion of a pilot symbol on seven symbols of time [10] [11] [12] [13], this is reflected by a loss of flow of $1 / 7$.

In summary, mobile applications always require higher bit rates and subscribers always demand better quality, fast and fast connection speeds, and secure mobility. It should be noted that each transition from one generation to another, a new access technique appears. The optimization of frequency resources and the improvement of the quality of services are two important factors to consider when switching from one access technique to another.

\section{LITERATURE REVIEW}

In this work, we are interested in the physical layer of the radio mobile system. We will introduce an intelligent method to improve channel estimation results. As we know SC-FDMA and OFDMA system are used from mobile generation, the first system for uplink (from mobile station to base station) and the second system for downlink (from base station to mobile station).

This project will present new adapted estimation techniques to both SC-FDMA and OFDMA system, all belonging to the class of techniques known as Data-Aided (DA)[14] or signal addition, but we will made experimentations only for downlink systems. For this category of estimators, the information used is a part of the transmitted signal located in the domain timefrequency and called learning sequence or references. Our principal aim is to introduce a new channel estimation technique to reduce the level of interference between sub-carriers of the channel (ICI) introduced in case of high mobility also we aim by using intelligent method to improve network throughput.

N. Samuel, T. Diskin, and A. Wiesel [15], had presented their paper (Deep mimo detection), This work deals with the detection by MIMO (Multiple-Input-Multiple-Output) antennas. The authors focused on the application of Deep Artificial Neural Networks. They began their work by presenting the features of Deep Learning and then they studied the different types of architectures of the neural networks that adapt to the task of estimation and detection of the 
channel. They studied the two cases of the MIMO systems namely the case of constant MIMO channel and the case where the parameters of the MIMO cabal are variable. Their work has been compared with the classical methods of transmission channel detection and estimation. They have provided that the deep learning can realize a good precision and present not only robustness but also low complexity.

In [16] The authors presented their works which consist of several new solutions that revolve around Deep Learning techniques for radio communication system physical layer. All presented techniques are at the base of learning. The communication system proposed by the authors is presented as being a self-encoder, they have developed a new fundamental way of thinking about the communication system of mobile networks. Their design is presented as an end-to-end reconstruction task for either uplink or downlink. Their principle aim was to achieve an optimization of the components of both transmission part and reception part in a single process. They applied techniques based on artificial convolutional neural networks (CNN) on raw IQ samples for modulation classification in order to achieve good accuracies and competitive results over traditional feature-based systems.

Hao Ye and all [17] have presented a paper called "Power of Deep for Channel Estimation and Signal Detection in OFDM Systems. The authors had presented results for channel estimation by using deep learning to detect signal in context of OFDM systems. Their work consist on handling wireless OFDM end-to end channel. Their approach consists of detecting the transmitted signal by using Channel State Information (CSI). As all kind of intelligent system, they used two stages to avoid channel distortion. The first stage consists of training offline using the data generated from simulation based on channel statistics and in the second stage they used obtained results for recovering the online transmitted data directly. Their results by simulation had shown the advantages of Deep Learning especially when the channel various rapidly and the distortion and diffraction are complicated.

Deep Learning-Based Channel Estimation had been diffused by Mehran S. and All [18]. In this work the OFDM matrix has been considered as image, so the two-dimensional time-frequency which represent the response of a fast fading communication channel had been handled as a twodimensional image. The mission of Deep learning is to find the channel unknown values by sending some pilot value called reference symbol. The authors had obtained highly competitive results compared to Minimum Mean Square Error algorithm.

The first work on adaptive channel monitoring was by K. Seshadri Sastry et al. [19] which began with the realization of an adaptive OFDM modulation system using Fuzzy Interference System FIS based on fuzzy logic. This interface makes possible improving the system capacity by maintaining a good quality of system and with playing on certain parameters. Adaptive modulation systems based on hardware have been shown to be ineffective in deciding on modifying or changing the modulation according to given conditions.

Previous research work has also shown that the use of fuzzy logic in the decision-making interface makes the system more efficient. The implementation of a method that enhances the capacity of the system in a Rayleigh channel context has been performed by Atta-ur-Rahman et al. [20]. However Harivikram and al. [21] studied the OFDM system with adaptive modulation and a coding rate and the results obtained are better than the normal OFDM system without adaptation. Faezah and al. [22] realized an adaptive OFDM transmission scheme. They also concluded that the adaptive transmission scheme is better than the fixed transmission system. 
The adaptation algorithm helps to improve throughput and increase BER performance. The work of K. Seshadri Sastryet and al. [23] revolved around the same principle. Qureshi and al. [24] proposed adaptive modulation and coding scheme using a fuzzy rule-based system where product codes are used with QAM modulation that increases the data rate in an OFDM system with a rate binary error and a fixed transmission power for each subcarrier. Atta-ur-Rahman Fuzzy and al. [20] proposed a system based on adaptive modulation with a coding scheme that shows that the use of fuzzy rules is more powerful in terms of error rate per bit. Khem Kumar et al. [25] proposed an adaptive OFDM modulation system using a FIS based on the bit error rate and modulation order of the QAM techniques. The system thus designed performs better than the conventional modulation system because of its adaptive nature. Parminder Kaur et al. [26] presented an OFDM system that provides high spectral efficiency using the fuzzy neuro approach.

In conclusion we can deduce that most of the research work done in the context of channel estimation is of two types: The research work mentioned in the first part is based on the classical channel estimation method such as the Least Square method and the MMSE method. The research works cited in the second part are a bit clever since they are based on a technique among the tools of artificial intelligence. Our work will be based on two intelligent tools assembled, namely Deep Learning and Fuzzy Logic, that's the reason we're talking about Hybrid Deep Learning. This hybridization will make it possible to exploit the advantages of the two tools and to benefit from their complementarity.

\section{THE EXPERIMENTAL MODEL}

We have chosen Matlab because this platform offers many essential possibilities by its toolboxes for system design. Matlab functionalities revolve around a language that allows software processing to analyze systems and the data derived from them. But it is also possible to represent these systems in graphical form with the module, or "toolbox", Simulink. In this one, rather than programming equations there, the latter are represented in graphical form, the block diagrams. Consequently, Matlab toolboxes are broken down into several categories: The first is the Matlab product family which provides code to insert into its own scripts; The second is Simulink family of products that uses graphical tools; The first family (Matlab products) includes more than sixty "toolboxes" covering the following areas: parallel computing, mathematics, statistics and optimization, system control, signal processing and wireless communications, processing image and artificial vision, tests and measurements, finance as well as computer biology, code generation, application deployment and access to databases as well as reporting (automated production of reports and reports).

This work deals with the downlink system whose access technique is OFDMA. Figure1 represents the transmitter system model.

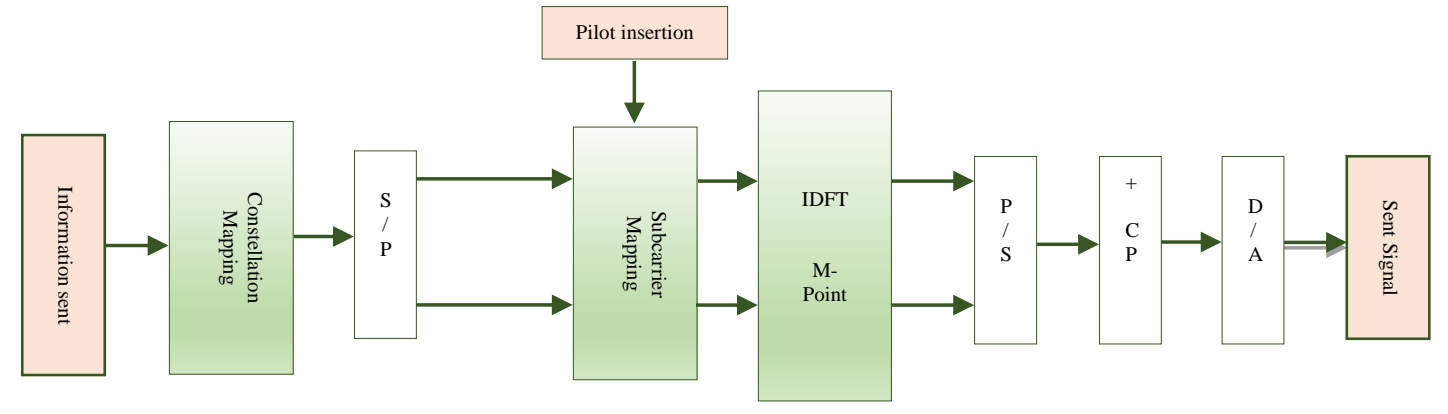

Figure 1. Transmitter system model 
We designed an OFDMA model for the transmission and reception. At the output of the source(figure 1), a binary message is generated randomly, its dual reception is estimated. The difference between the two makes possible to calculate the Binary Error Rate. The second component of the chain is the channel coding whose output is a coded binary message, its dual at the reception is estimated. Errors between these two signals are corrected by channel coding. Then we have the Mapping to obtain a vector of complex symbols of a constellation $\Omega$ of size K such as a Phase Modulation MDP4 or an Amplitude Modulation in Quadrature MAQ 64. Then the serial/parallel converter: The vector of complex symbols of useful data paralleled on different sub-carriers. The representation in the frequency domain of the impulse response of the transmission channels is a sum of sinusoids. We study the simulated signal $U(t)$ over a period $T s$ corresponding to $\mathrm{N}$ samples. $\mathrm{U}(\mathrm{t})$ is the resultant of a Dirac pulse $\delta(t)$ emitted through a channel with $L$ paths and is defined by:

$$
U(t)=\sum_{\beta=0}^{L} h_{\beta} \delta\left(t-\tau_{\beta}\right)
$$

with $\boldsymbol{h}_{\boldsymbol{\beta}}$ a random variable according to a centered Gaussian law.

First, the transition to the frequency domain is performed by applying an FFT to this response, we obtain the sum of sinusoids representatives of the transmission channel. Then, to make the experiments relating to the comparison of different interpolation methods already mentioned, we take some well-chosen values of the frequency response: These values will act as pilots. Then we perform the interpolation using the specific interpolation algorithms, in order to obtain an estimate of the sum of sinusoids from the pilots values. Finally, we calculate the BER between the values of the frequency response estimated by the interpolation algorithms on one side and the exact value on the other side.

We find in the literature a set of channel models that can play the role of the air interface. These channels are required to perform simulations, tests and verifications of transmission and radio reception between the mobile station and the base station "eNode B." We performed the simulation work on the channel model entitled: Multipath Fading Propagation Conditions (MFPC): Specifying the Three Delay Profiles: Extended Pedestrian Model (EPA), Extended Vehicular Model (EVA), and Extended Typical Urban Model (ETU). Delay profiles represent the low, medium, and high for the propagation environment. The multipath delay profiles for these channels are shown in the following tables:

Table 1. Characteristics of the models of the channels used

\begin{tabular}{|c|c|c|c|c|c|}
\hline \multicolumn{2}{|c|}{ 1. EPA } & \multicolumn{2}{c|}{ 2.EVA } & \multicolumn{2}{c|}{ 3. ETU } \\
\hline Delay (ns) & Attenuation(dB) & Delay (ns) & Attenuation (dB) & Delay (ns) & Attenuation(dB \\
& & & & 0 & -1.0 \\
\hline 0 & 0.0 & 0 & 0.0 & 50 & -1.0 \\
\hline 30 & -1.0 & 30 & -1.5 & 120 & -1.0 \\
\hline 70 & -2.0 & 150 & -1.4 & 200 & 0.0 \\
\hline 90 & -3.0 & 310 & -3.6 & 230 & 0.0 \\
\hline 110 & -8.0 & 370 & -0.6 & 500 & 0.0 \\
\hline 190 & -17.2 & 710 & -9.1 & 1600 & -3.0 \\
\hline 410 & -20.8 & 1090 & -7.0 & 2300 & -5.0 \\
\hline & & 1730 & -12.0 & 5000 & -7.0 \\
\hline
\end{tabular}


All paths mentioned in the previous tables have specific Doppler effects, in addition to multipath delay profiles.

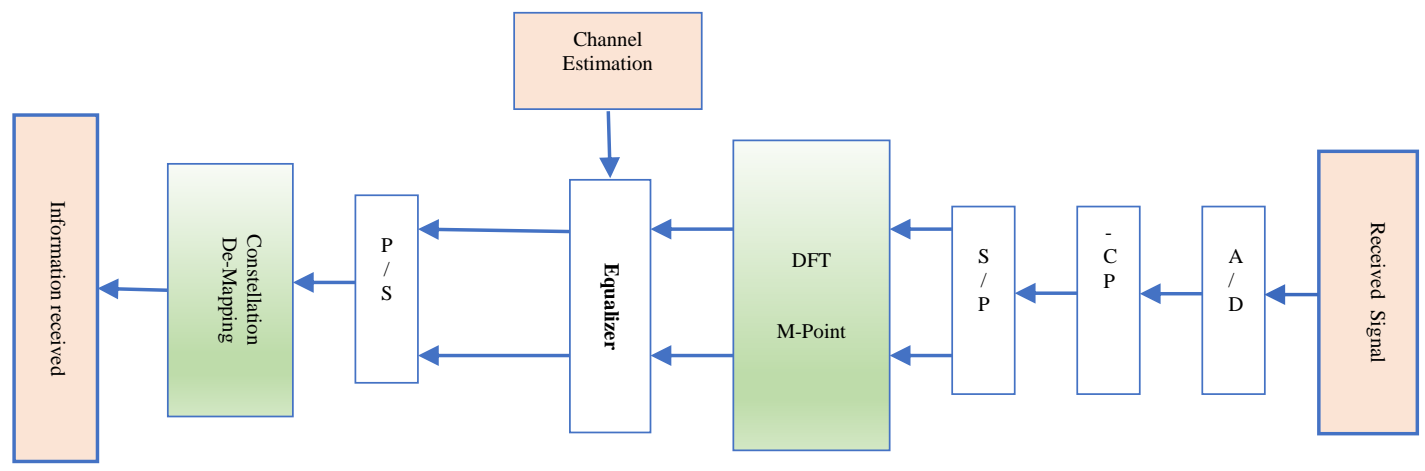

Figure 2. Receiver system model

As in transmitter we start by randomly generating information through Matlab then we apply the modulation technique according to the standard. the signal emitted will be sent in one of three models of multipath channel for which we add an Additive White Gaussian Noise, in the reception we apply the inverse of the operations made by the transmitter as shown in figure 2 .

The first advantage of using the parallel transmission technique over several orthogonal subcarriers is the increase spectral efficiency. This solution given by the OFDM to current generations of mobile radio communication systems. However, the channel estimation requires insertion of pilot symbols in the grid of the transmitted data. In most cases, the channel impulse response estimation operation is performed by interpolating the received channel parameters using the pilot symbols to estimate the rest of the data grid. The higher the number of pilot symbols in the grid, the better will be the result of the interpolation, but in return we record a greater loss in the flow rate. Several research works have focused on this topic to find a compromise between the distribution of inserted pilot symbols and spectral efficiency.

Table 2. System parameters

\begin{tabular}{|c|c|}
\hline Parameter & Value \\
\hline Number of transmitted bits & 128000 \\
\hline Modulation & BPSK/QPSK \\
\hline OFDM Subcarriers & $64 / 128$ \\
\hline Number of pilots $(\mathrm{Np})$ & $8,16,32$ \\
\hline Cyclic prefix length $(\mathrm{Lcp})$ & 16 \\
\hline Carrier Frequency $(\mathrm{fc})$ & $2.4 \mathrm{Ghz}$ \\
\hline
\end{tabular}

Throughout this work, we focus on the implementation constraints of a classical and hybrid neuron network in a channel estimation system in an OFDM context. We will present a series of experiments made on the classical different systems proposed. We will start by presenting the simulation results of two classical estimation methods namely LSE and MMSE. Results will be compared to the theoretical study to prove the difference in performance between LSE and MMSE. 


\section{SiMULATIONS OF LSE AND MMSE ESTIMATORS}

The objective of the least square criterion is to minimize the cost function $\mathrm{F}$ which is defined as the square of the standard of the error vector. The error vector is obtained by calculating the difference between the received signal vector $\mathrm{Y}$ and the product of the transmitted signal vector $\mathrm{X}$ with a diagonal matrix $\mathrm{M}$ whose coefficients are assumed to be optimal. The estimate is given by the following equation:

$$
\widehat{L S E}=\underline{M}_{\text {optimale }}
$$

The cost function is given by:

$$
F_{L S E}=|Y-\underline{M} X|^{2}
$$

The signal received is the sum of the signals on each of the subcarriers from 0 to $\mathrm{P}-1$

$$
F_{L S}=\sum_{i=0}^{P-1}\left|Y_{i}-M_{i} X_{i}\right|^{2}
$$

We are in front of a vector of $\mathrm{P}$ lines. To seek the minimum of the function $F_{L S E}$ We compute the derivative $D_{i}$ of each value of the line of this vector compared to the variable $\mathrm{M}$. Then we solve for each value of $i$ the equations $D_{i}=0$.

$$
D_{i}=-2 E_{i}\left(Y_{i}-M_{i, o p t} X_{i}\right)
$$

The estimate of all the elements of the vector will be given by:

$$
\widehat{L S E_{i}}=\frac{Y_{i}}{E_{i}}=H_{i}+\frac{W_{i}}{X_{i}}
$$

So for each value of $\mathrm{i}$, we can write an estimate of the subcarrier $\mathrm{i}$ :

$$
\widehat{L S E}=Y \underline{X}^{-1}=H+W \underline{X}^{-1}
$$

It is obvious from expression (7) that the least square estimator is sensitive to the noise level. The higher the noise introduced by the propagation channel, the more the estimator will be affected. Several researchers have focused their work on the lack of noise sensitivity presented by the estimator at least squares because the LSE cannot offer acceptable results for frequency response estimation of the transmission channel. The task performed by LSE has two drawbacks, the first is that the estimate signal is noisy and the second is the need for an interpolation method to estimate all the data received in the grid time/frequency. In addition, the second estimation technique which concerns the estimator with a criterion for minimizing the quadratic mean error MMSE, has shown better performance than LS estimator. The advantage of the MMSE is that it can play the role of the interpolator filter and its major drawback is the high complexity.

We will now introduce the minimum mean square error criterion (MMSE). This method, which is based on the criterion of minimum mean square error (MMSE), takes into account the correlation of the channel, which makes it more precise than the first method. This method presents two major difficulties, the first is due to the complexity of its implementation, since two independent filters are applied successively. The second difficulty consists in computation starting from the knowledge of certain properties of the paths of the channel (Doppler frequency, maximum spreading of the subchannels). It is obvious that the performance of the MMSE 
estimator is much more efficient than the LS estimator in particular in the case where the signal to noise ratio is low. The goal of the MMSE method is to minimize the cost function defined by the mean square error of the vector $H-\underline{M} Y$.

.And $\mathrm{M}$ is the matrix whose coefficients must be optimized such that:

$$
F_{M M S E}=E\left\{\|H-\underline{M} Y\|_{F}^{2}\right\}
$$

The channel estimate will be obtained by

$$
\widehat{M M S E}=\underline{M}_{o p t} Y
$$

And that the signal received is given by: $Y=H \underline{X}+W$ and we assume that $\mathrm{H}$ and $\mathrm{W}$ are two uncorrelated vectors and of zero mean.

We can develop (9) to obtain:

$$
\begin{aligned}
F_{M M S E}=\boldsymbol{E}\left\{\|H-\underline{M} Y\|_{F}^{2}\right\} & =E\left\{\operatorname{tr}(H-\underline{M} Y)(H-\underline{M} Y)^{H}\right\} \\
= & \operatorname{tr}\left(\boldsymbol{E}\left\{H H^{H}-H Y^{H} \underline{M}^{H}-\underline{M} Y H^{H}+\underline{M} Y Y^{H} \underline{M}^{H}\right\}\right)
\end{aligned}
$$

We inject the expression $Y=H \underline{X}+W$ in (10), we obtain:

$$
\begin{aligned}
F_{M M S E}= & \boldsymbol{E}\left\{\operatorname{tr}\left(H H^{H}\right)\right\}-\boldsymbol{E}\left\{\operatorname{tr}\left(H(H \underline{X}+W)^{H} \underline{M}^{H}\right)\right\}-\boldsymbol{E}\left\{\operatorname{tr}\left(\underline{M}(H \underline{X}+W) H^{H}\right)\right\}+\boldsymbol{E}\left\{\operatorname{tr}\left(\underline{M}(H \underline{X}+W)(H \underline{X}+W)^{H} \underline{M}^{H}\right)\right\} \\
= & \operatorname{tr}\left(\underline{\boldsymbol{R}}_{H}\right)-\operatorname{tr}\left(\underline{\boldsymbol{R}}_{H} \underline{X}^{H} \underline{M}^{H}\right)-\operatorname{tr}\left(\underline{\boldsymbol{R}}_{H} \underline{X M}\right)+\operatorname{tr}\left(\underline{M}\left(\underline{X \boldsymbol{R}_{H}} \underline{X}^{H}+\sigma^{2} \underline{I}\right) \underline{M}^{H}\right)
\end{aligned}
$$

In order to obtain the optimal value of the matrix (M): $\underline{M}: \underline{M}_{\text {opt }}$, we make the derivative of the expression (11) with respect to $\underline{M}$ and we seek for which value of $\underline{M}$ is equal to zero, $\left(\frac{\partial F_{M M S E}}{\partial \underline{M}}=0\right) . \frac{\partial F_{M M S E}}{\partial \underline{M}}=0-\underline{\boldsymbol{R}}_{H} \underline{X}^{H}-\left(\underline{X \boldsymbol{R}_{H}}\right)^{H}+2 \underline{M}\left(\underline{X \boldsymbol{R}_{H}} X^{H}+\sigma^{2} I\right)$

Knowing that the covariance of the channel $\underline{R}_{H}$ is Hermitian, is identical to its conjugate $\underline{R}_{H}=\underline{R}_{H}^{H}$. We can write $\underline{M}_{\text {opt }}$ :

and

$$
\begin{aligned}
\underline{M}_{\text {opt }} & =\frac{\underline{R}_{H} \underline{X}^{H}}{\left(\underline{R^{\prime}} H \underline{X}^{H}+\sigma^{2} I\right)} \\
\underline{M}_{\text {opt }} & =\underline{R}_{H} \underline{X}^{H}\left(\underline{X \boldsymbol{R}_{H}} \underline{X}^{H}+\sigma^{2} I\right)^{-1}
\end{aligned}
$$

$$
\underline{M}_{o p t}=\underline{\boldsymbol{R}}_{\boldsymbol{H}}\left(\underline{\boldsymbol{R}}_{H}+\underline{(X}^{H} \underline{X}\right)^{-1} \sigma^{2} \underline{I^{-1} X^{-1}}
$$

So we can extract the expression from the LMMSE channel estimator:

$$
\begin{aligned}
\widehat{H}^{L M M S E}=\underline{M}_{o p t} \cdot R \widehat{H}^{L M M S E} & =\underline{\boldsymbol{R}}_{\boldsymbol{H}}\left(\underline{\boldsymbol{R}}_{H}+\left(\underline{X}^{H} \underline{X}\right)^{-1} \sigma^{2} \underline{I}\right)^{-1} X^{-1} \cdot R \\
\widehat{\boldsymbol{H}}^{L M M S E} & \left.=\underline{\boldsymbol{R}}_{\boldsymbol{H}}\left(\underline{\boldsymbol{R}}_{H}+\underline{(X}^{H} \underline{X}\right)^{-1} \sigma^{2} \underline{I}\right)^{-1} \widehat{\boldsymbol{H}}^{L S}
\end{aligned}
$$




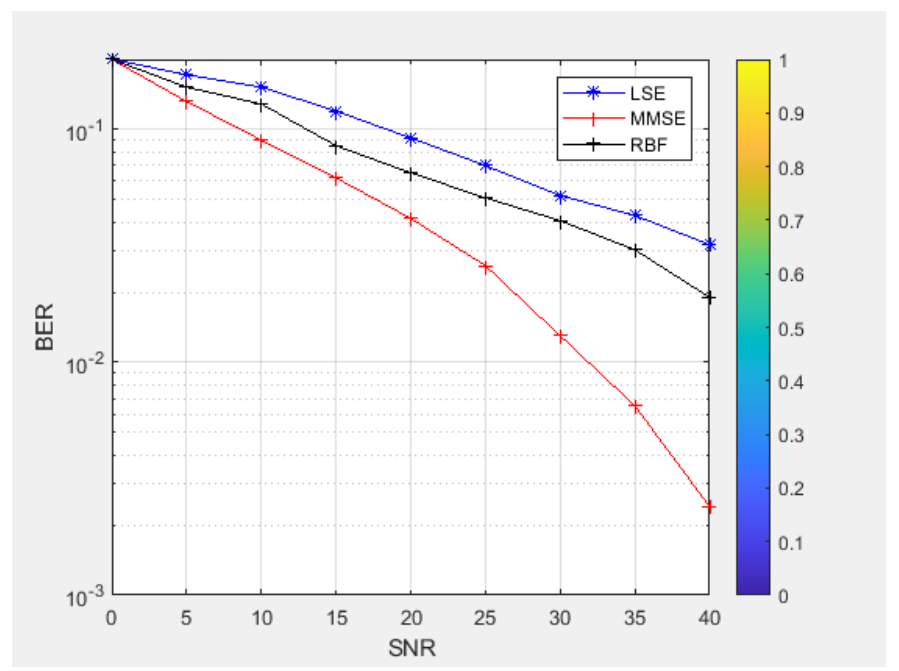

Figure 3. Comparison of the performances of LSE and MMSE estimators

It is obvious that the complexity is much more than that of the classical least square method. This complexity is due to the matrix inversion operation and the multiplication operations. But the LMMSE estimator does not have only disadvantages, since it is less sensitive to noise than the LS de fact that it is based on the mean square error and the Gaussian noise being at zero mean. This estimator can also be effective in the case of interpolation, it will be used in the case where the pilot symbols are weakly distributed in the frame.

We have simulated using Matlab the behavior of the LS and MMSE simulators, taking the case of 128 subcarriers and QPSK modulation. Figure 3 illustrates the difference in performance of these two estimators in an OFDMA context. The simulation results confirm the theoretical study. Figure 3 shows that the MMSE estimator performs better than the LSE estimator.

\section{CONDition OF NeURAL Networks IMPlementation}

Artificial intelligence is one of the specialties of computer science that revolves around the simulation of human behavior and the modeling of the brain of the human being. The first networks of artificial neurons (RNA) was proposed by Mc Culloch [27] (neurophysiologist) and Pitts (logician) in 1943 [28]. Sixteen years after work by Rosenblatt in 1959 to present more advanced simulations, he introduced the concept of input layer and output layer and applied it to simulate the human retinal mechanism for forms recognition. The year 1970, the first connectionist neural network has not been successful due to hardware and software constraints for this period. Afterwards, the symbolic or sequential approach has been introduced to promote expert systems. The goal was to use the qualities of the human brain to give automation to the machine. To do so, three elements are introduced namely, a knowledge base, a facts and observation base and an application mechanism. The knowledge base groups all the knowledge of human experts in the form of basic or more elaborate logical propositions using quantifiers (first-order logic). The fact base contains the observations according to the model or the case to be treated and an inference engine responsible for applying the expert rules on the basis of facts in order to deduce new facts until the achievement of a goal, already determined. 


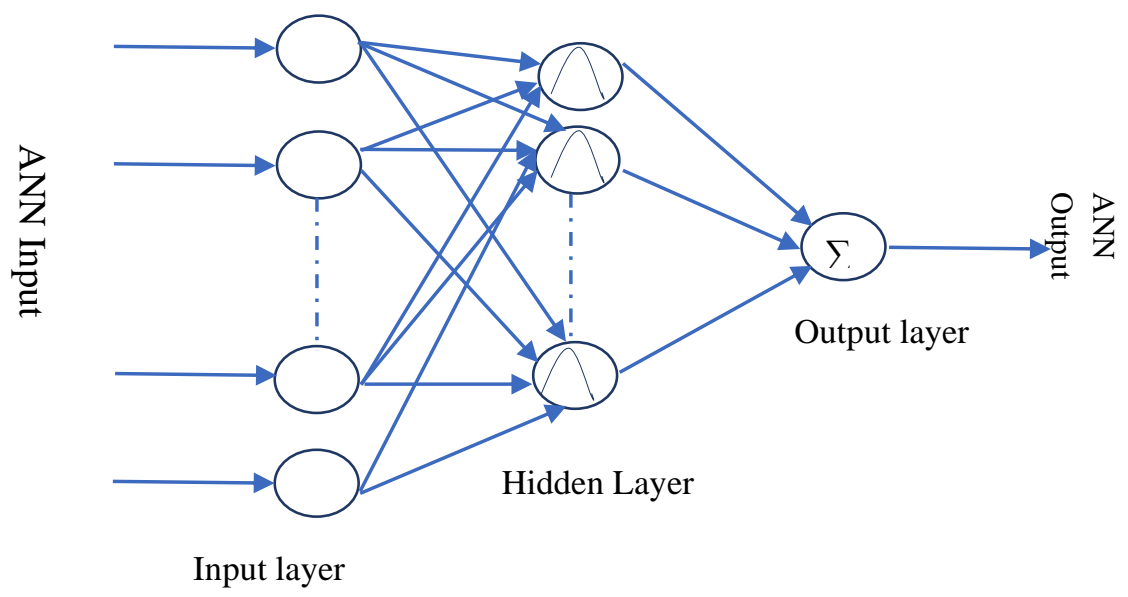

Figure 4. Artificial Neural Networks: Radial Base Function

A neuron network is a set of layers formed by neurons. The complexity of a neuron network differs according to the nature of the neurons, the architecture, and the number of layers and the presence of feedback loop. The literature distinguishes two major classes of neural networks, the symbolic neural networks and the connectionist neural networks. In this work, we studied very specific neural networks. They are networks of neuro-symbolic neurons that are characterized by a speed of convergence to perform well the task of estimating the channel. The neuro-symbolic neuron network, also called hybrid, is a network characterized by a module for inserting fuzzy rules.

We have introduced a neural network interpolation system and made a comparison with the different classical techniques. The use of the neural solution imposes the respect of several criteria: the treatment of complex numbers, the choice of the topology of the network and the location of the network in the chain of transmission and several other factors. Multilayer topology is often chosen in the literature. The choice of architecture takes into consideration the complexity factor. In fact, more neurons in the network translates into more complexity. The choice of transfer functions is made according to the problem. Two types of networks have been confirmed as universal approximators namely Radial Base Function (RBF) networks and Perceptron Multi Couches (PMC) networks. We have used in this paper RBF as a neural interpolation solution, the results are shown in figure 5 .

The RBF network model was introduced in 1985 by Powel [37], It is a supervised neural network. The neurons in the hidden network layer are generally Gaussian functions. The topology of the RBF network is based on only three layers: The input layer which retransmits the inputs without any modification, the hidden layer formed by the Gaussian neurons and an output layer whose neurons are mostly active by a linear function. It should be noted that the layers of the RBF network have no connection within the same layer. Each layer has a connection with the next layer. The basic difference between neural networks with perceptron topology and RBF neural networks is the activation function, since the activation functions of RBF networks are Gaussian centered on a point in the input space and those of the perceptron are sigmoid. For an RBF neural network input, the height of the Gaussian represents the output of the RBF neuron.

We applied an RBF network with a Block pilot arrangement, this type of arrangement is suitable for a slow fading channel. The simulations were carried out on two dimensions. 
The spacing between the pilot symbols is $\mathrm{K}$ in the time domain and $\mathrm{L}$ in the frequency domain. A time/frequency matrix having traversed the OFDM chain,

Let us note by $I_{p}$ the index of all the subcarriers reserved for the pilot symbols. These pilots are uniformly distributed in the matrix:

$$
X(k)=\left\{\begin{array}{cl}
p_{k} . & \text { if } k \in I_{p} \\
d_{k}, & \text { else }
\end{array}\right.
$$

The estimation of the channel at the pilot symbol level is given by applying the method of least squares:

$$
\widehat{H}(k)=\frac{X(k)}{Y(k)}
$$

Then, an interpolation based on the RBF network is used to represent the frequency response of the channel to all the subcarriers.

The channel response is given by

$$
\begin{aligned}
& \widehat{H}(l)=\sum_{k \in I_{p}} w(k) \varphi_{k}(l) \\
& =\sum_{k \in I_{p}} w(k) \varphi_{k}\left(\frac{\left|n-\mu_{k}\right|^{2}}{\sigma_{k}^{2}}\right)
\end{aligned}
$$

With $\varphi_{k}(x)$ is the Gaussian function and the terms $\mathrm{w}(\mathrm{k})$ are the weights of the neurons of the output layer. Figure 5 illustrates the improvement made by an RBF neural network to the channel estimation process.

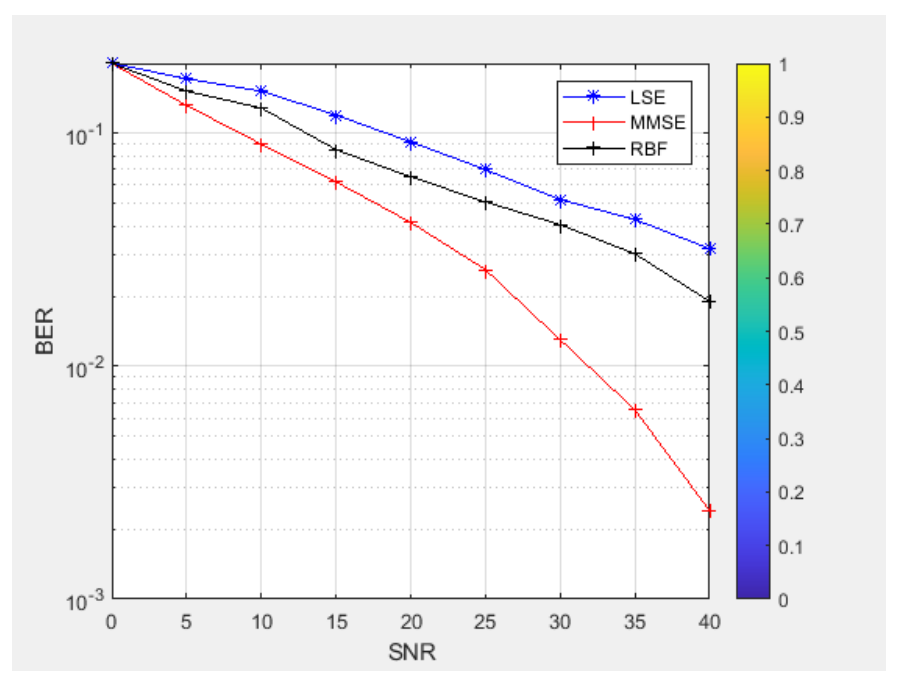

Figure 5. Comparison of the performances of RBF with LSE and MMSE estimators

The introduction of neural networks has provided new forms of interpolation and approximation, effective compared to classical techniques. For standard estimation techniques, the interpolator is usually linear or cubic, its intervention consists of approximating the data received from the reference data. The neural networks, which offer better results by interpolating with minimum of received pilot symbols and by following the variations of channel propagation. Figure 5 shows that interpolation by a classic neural network based on Radial Base Function can improve the 
quality of estimation relative to LSE but may not reach the quality offered by MMMSE. However, from the point of view complexity Radial Base Function is better than MMSE. Most neural network estimators have the disadvantage of slowness in terms of convergence. The speed of convergence of the neural network depends essentially on the number of nodes and the numbers of the layers [29]. One of the solutions that can improve the speed of convergence is the initialization of the starting weights of the neural network. The "insertion" function which we will present in this project will solve the problem of slowness during neural learning.

Neural networks can manipulate complex numbers as input and output parameters and are able to set complex decision areas with nonlinear decision boundaries [32]. In addition, and due to the non-linear characteristics of neural networks, and their specific architectures, they have been successful in the area of channel estimation and equalization [30] [31] [32] [33]. One of the first applications of neural networks for digital communication channel equalization has been made by Siu et al. [34]. Neural networks have been proposed as a channel estimator with use of QAM and QPSK modulation, respectively [35] and [36]. In [37], a three-layer neural network was used as a channel prediction tool for MIMO systems.

\section{DEEP LEARNING:}

Deep Learning (DL) is a type of artificial intelligence derived from machine learning where the machine is able to learn by itself, unlike programming where it is content to execute rules to the letter predetermined. DL is based on a network of artificial neurons inspired by the human brain.

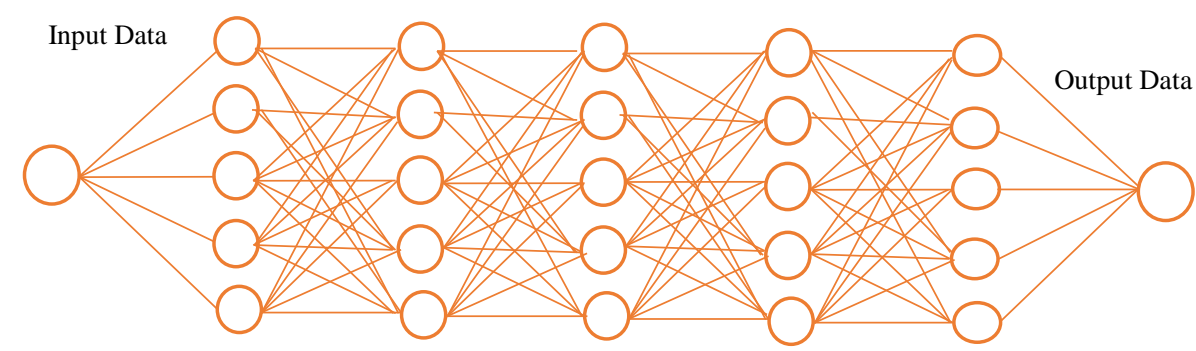

Figure 6. Deep Learning example

This network is made up of tens or even hundreds of "layers" of neurons, each receiving and interpreting information from the previous layer. For example, the system will learn to recognize letters before tackling words in a text or determine if there is a face in a photo before finding out who it is. Deep Learning is used in many fields: image recognition, automatic translation, medical diagnosis, automatic moderation of social networks, financial prediction and automated trading, chatbots (conversational agents), space exploration, intelligent robots.

\section{FuZZY LOGIC}

Fuzzy logic is a method proposed by Lotfi Zadeh in fuzzy set theory in 1965 [47] [48]. Fuzzy logic has been applied in several areas. Unlike the Boolean logic where the truth values of the variables can only be 0 or 1 , fuzzy logic is a form of multiple logical values in which the truth values of the variables can be any real number between zero and one. The Fuzzy logic has been extended to manipulate the concept of partial truth, where the truth value may vary between quite true and completely false. In addition, when linguistic variables are used, these propositions can be managed by specific functions. An important part of our work is to establish and insert fuzzy rules into the classical neural network. This contribution consists of constructing a hybrid neural 
network capable of rapidly converging and giving better results than conventional one, and applying it in channel estimation and interpolation algorithms.

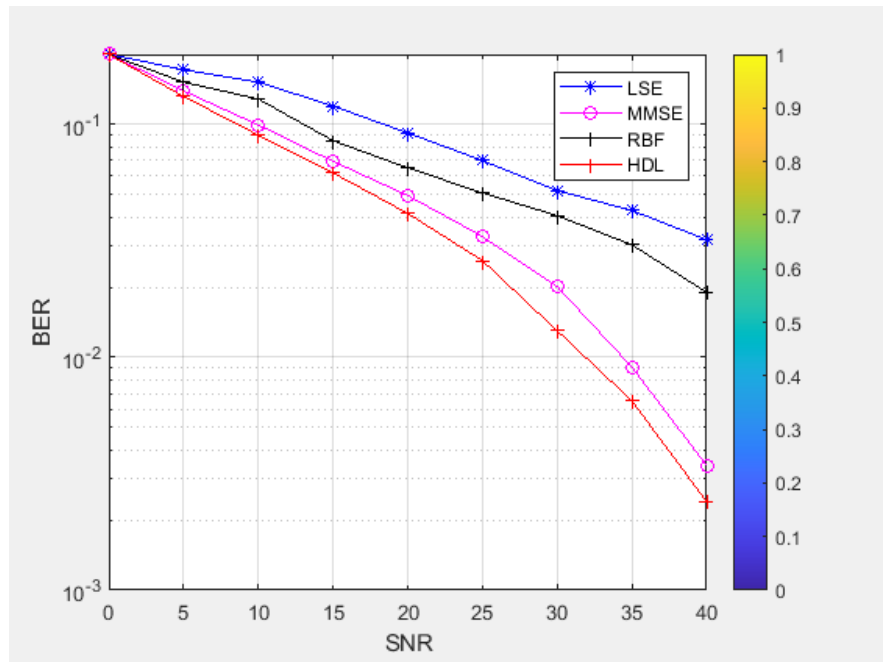

Figure 7. Comparison of the performances HDL with ANN, LSE and MMSE estimators

The mobile user has three different types of channels. depending on his mobility, he will be called upon to use the appropriate channel. Since the deep neural network always begins its learning phase with a random initialization of these parameters, we opted for the use of fuzzy logic in order to fix these initialization parameters. Setting the parameters at the beginning allows the network to converge quickly. Figure 7 shows that the combination of deep neural network theory with the fuzzy logic method HDL has made it possible to improve the quality of interpolation in comparison with the MMSE. HDL can reduce the number of pilot symbols inserted in order to improve the throughput.

\section{CONCLUSiON}

This work has two main objectives. The first objective is to present a new hybrid deep learning model based on fuzzy rules that can be applied in several domains. The second objective of this work is to propose new ways to improve the spectral efficiency of next generation mobile communications systems, without using additional resources in terms of bandwidth and with the minimum complexity of the proposed systems.

In $4 \mathrm{G}$, channel estimation is done in a supervised manner. This supervision is ensured by periodically inserting pilot symbols into the data frame. The insertion of a symbol concerns all the frames and is done every seven symbols, which will cause a loss of 1/7 of the flow. We used in this work an intelligent system based on hybrid neural networks and fuzzy logic to reduce the loss of flow. We can avoid the insertion of pilot symbols by improving the performance of conventional neural networks.

We dedicated the first section of our experimentations to study classical estimators and to present the results of the simulations of LSR and MMSE we have presented the necessary analyzes and comparisons. The second contribution concerns the area of smart channel estimation. It consists in proposing a new intelligent interpolation method making it possible to improve the spectral efficiency of the future generation mobile system. Hybrid Deep Learning is presented in this work as approximation and interpolation techniques that: 
-Performs the interpolation with the minimum possible pilot symbols, thus increasing the bit rate of the link.

-Follows the state of channel variation

-Ensures rapid convergence with minimal complexity

\section{REFERENCES}

[1] Widrow G et Holff M. Adaptive switching circuits. In Wester Electronic Show and Connection, Connection record, volume 4. page 96-104, Institue of Radio Engineers, 1960.

[2] Monserrat, J. F., Mange, G., Braun, V., Tullberg, H., Zimmermann, G., \& Bulakci, Ö. (2015). METIS research advances towards the 5G mobile and wireless system definition. EURASIP Journal on Wireless Communications and Networking, 2015.

[3] Hämäläinen, S., Sanneck, H., \& Sartori, C. (2012). LTE self-organising networks (SON): network management automation for operational efficiency. John Wiley \& Sons.

[4] Ponulak, F., \&Sinyavskiy, O. (2015). U.S. Patent No. 8,943,008. Washington, DC: U.S. Patent and Trademark Office.

[5] J. Palicot and Y. Louet. Power ratio definition and analysis in single carrier modulation. European Signal Processing Conference, EUSIPCO, 2005.

[6] J. Tellado-Mourelo. Peak to Average Power Reduction for Multicarrier Modulation. PhD thesis, Stanford University, Sept 1999.

[7] D. Guel, Y. Louet, and J. Palicot. A geometric method for papr reduction in a signal adding context for ofdm signals. In Proc. 15th Int Digital Signal Processing Conf, pages 347-350, 2007.

[8] Genevieve Baudoin. Radiocommunications Numériques : Principe, Modélisation et Simulation (Tome 1). 2002.

[9] A. Samukic. UMTS Universal Mobile Telecommunications System : development of standards for the third generation. In Proc. Bridge to Global Integration.IEEE Global Telecommunications Conf. GLOBECOM 98, volume 4, pages1976-1983, 1998.

[10] I. Poole. What exactly is cdma 2000 ? Communications Engineer,4(1) :46-47, 2006.

[11] Youngchul Sung, Yirang Lim, Lang Tong, and A.-J. van der Veen. Signal processing advances for $3 \mathrm{~g}$ wcdma : From rake receivers to blind techniques.47(1):48-54, 2009.

[12] Channel estimation in OFDM systems utilizing pilots, number 187-190, Finland, September 1996. IEEE Nordic Signal processing Symposium.

[13] Sosthène Yameogo, Jacques Palicot, and Laurent Cariou. Channel estimation technique by hidden signal : Application in the sc-fdma context. ICWMC, 2010.

[14] H. Ye, G. Y. Li, and B.-H. Juang, (Power of deep learning for channel estimation and signal detection in ofdm systems) IEEE Wireless Communications Letters, vol. 7, no. 1, pp. 114-117, 2018.

[15] Neev Samuel, Tzvi Diskin, Ami Wiesel "Deep MIMO detection" 2017 IEEE 18th International Workshop on Signal Processing Advances in Wireless Communications (SPAWC) December 2017 [9] [16] Timothy O'Shea ; Jakob Hoydis "An Introduction to Deep Learning for the Physical Layer" IEEE Transactions on Cognitive Communications and Networking (Volume: 3 , Issue: 4, Dec. 2017 ) DOI: 10.1109/TCCN.2017.2758370DOI: 10.1109/SPAWC.2017.8227772

[17] Hao Ye ; Geoffrey Ye Li ; Biing-Hwang Juang "Power of Deep for Channel Estimation and Signal Detection in OFDM Systems" IEEE Wireless Communications Letters ( Volume: 7 , Issue: 1, Feb. 2018 ) DOI: 10.1109/LWC.2017.2757490

[18] Mehran S., Vahid P.,Mirzai A., Sheikhzadeh H. "Deep Learning-Based Channel Estimation” IEEE Communications Letters ( Volume: 23 , Issue: 4, April 2019 ) 10.1109/LCOMM.2019.2898944

[19] K. Seshadri Sastry and Dr.M.S.Prasad Babu. "Adaptive Modulation for OFDM system using Fuzzy logic interface”, IEEE ICSESS, 368-371, 2010.

[20] Atta-ur-Rahman, Ijaz Mansoor Qureshi, and Aqdas Naveed Malik, "Adaptive Resource Allocation in OFDM Systems Using GA and Fuzzy Rule Base System". World Applied Sciences Journal 18.6, 836-844, 2012.

[21] Harivikram, T. S., et al. "Adaptive Modulation and Coding Rate for OFDM Systems". International Journal of Emerging Technology and Advanced Engineering. Vol. 3, Issue 2, 250-255, 2013.

[22] Faezah, J., and K. Sabira. "Adaptive Modulation for OFDM Systems" International Journal of Communication Networks \& Information Security 1.2, 2009. 
[23] K. Seshadri Sastry and Dr.M.S.Prasad Babu. "Fuzzy logic based Adaptive Modulation Using Non Data Aided SNR Estimation for OFDM system". International Journal of Engineering Science and Technology, Vol. 2(6), 2384- 2392, 2010.

[24] Qureshi, Ijaz Mansoor, and M. Zeeshan Muzaffar. "Adaptive Coding and Modulation for OFDM Systems using Product Codes and Fuzzy Rule Base System”. 54 Fuzzy Logic Based Modified Adaptive Modulation Implementation for Performance Enhancement in OFDM Systems Copyright (C) 2016 MECS I.J. Intelligent Systems and Applications, 2016, 5, 49-54 International Journal of Computer Applications 35.4, 2011.

[25] Khem Kumar Nagar, Kanchan Sharma. "Adaptive OFDM Implementation Using Fuzzy Inference System" International Journal of Advanced Research in Computer Science and Software Engineering, Volume 3, Issue 7, 2013.

[26] Parminder Kaur, Kuldeep Singh, Hardeep Kaur. "Performance analysis of MIMO-OFDM system in 4g technologies: A Review". International Journal of Mathematical and Engineering sciences, 2014.

[27] McCulloch, W. and Pitts, W.. A logical calculus of the ideas immanent in nervous activity. Bulletin of Mathematical Biophysics, 5:115-133, 1943.

[28] McCulloch, W. and Pitts, W.. A logical calculus of the ideas immanent in nervous activity. Bulletin of Mathematical Biophysics, 5:115-133, 1943.

[29] Mehran S., Vahid P.,Mirzai A., Sheikhzadeh H. "Deep Learning-Based Channel Estimation" IEEE Communications Letters ( Volume: 23 , Issue: 4 , April 2019 ) 10.1109/LCOMM.2019.2898944

[30] W. Weibull, "A Statistical Distribution Function of Wide Applicability". Journal of Applied Mechanics, vol. 18, pp. 293-297, 1951.

[31] Widrow G et Holff M. Adaptive switching circuits. In Wester Electronic Show and Connection, Connection record, volume 4. page 96-104, Institue of Radio Engineers, 1960.

[32] Rosemblat F. The perceptron : a probabilistic model for information storage and organistation in the brain. Psychological review, 65,1958.

[33] A. van Zelst, "Per-antenna-coded schemes for mimoofdm," Telecommunications Techno-logy and Electromagnetics Group Eindhoven University of Technology, 2003.

[34] Siu, S., Gibson, G. J., \& Cowan, C. F. N. (1990). Decision feedback equalization using neural network structures and performance comparison with standard architecture. Proceedings of the Institution of Electrical Engineers, 137(pt. 1), 221-225.

[35] Patra, J. C., Pal, R. N., Baliarsingh, R., \& Panda, G. (1999). Nonlinear channel equalization for QAM signal constellation using artificial neural networks. IEEE Transactions on Systems, Man, and Cybernetics, 29(2), 254-262

[36] M. J. D. Powell. Radial basis functions for multivariable interpolation: a review. In J. C. Mason and M. G. Cox, editors, Algorithms for Approximation. Clarendon Press, Oxford, 1987. 55

[37] M.J.D. Powell. The theory of radial basis functions approximation in 1990. In W.A. Light, editor, Advances in Numerical Analysis Volume II: Wavelets, Subdivision Algorithms and Radial Basis Functions, pages 105\{210. Oxford University Press, 1992.

[38] SMIRANI L., BOULAHIA J. and BOUALLEGUE R. "Artificial Neural Network Interpolation for SC-FDMA Channel Estimation" Revue enligne Wireless 2016 http://wirelesscommunication.conferenceseries.com/speaker/2016/lassaad-smirani-university-ofcarthage-tunisia-944256783

[39] SMIRANI L. and BOULAHIA J.Neural rule extraction: more precision in learning. WSEAS International Conference on Neural Network LISBON 2014.

[40] SMIRANI L. and BOUALLEGUE R. "An Uplink LTE-A Channel Estimation Method Based On Connexionist System" Energy and Manufacturing Engineering (ICAEME'2015 Jan. 7-8, 2015 at Dubai (UAE).

[41] SMIRANI L and BOULAHIA J . A New Channel Estimation Method for LTE-Advanced Uplink . ICMCE 2015 : XIII International Conference on Materials and Chemical Engineering. January 26 27, 2015 . JEDDAH.

[42] BOULAHIA J. and SMIRANI L. Experiments of Neuro Symbolic Hybrid Learning System With Incomplete Data" International Conference on Recent Advances in Engineering and Technology (ICRAET-2015), Feb 2015 Istambul, Turkey.

[43] SMIRANI L., BOULAHIA J. and BOUALLEGUE R "A semi blind channel estimation method based on hybrid neural networks for uplink LTE-A" International Journal of Wireless \& Mobile Networks (IJWMN) Vol. 8, No. 3, June 2016. http://aircconline.com/ijwmn/V8N3/8316ijwmn05.pdf 
International Journal of Wireless \& Mobile Networks (IJWMN) Vol. 12, No. 3, June 2020

[44] SMIRANI L. and BOULAHIA J.Neural rule extraction: more precision in learning. WSEAS International Conference on Neural Network LISBON 2014.

[45] SMIRANI L. and BOUALLEGUE R. "An Uplink LTE-A Channel Estimation Method Based On Connexionist System” Energy and Manufacturing Engineering (ICAEME'2015 Jan. 7-8, 2015 at cDubai (UAE).

[46] S. W. Tan C. F. Kwong, T. C. Chuah. The anfis handover trigger scheme: The long term evolution (lte) perspective. IEEE International Conference on Fuzzy Systems, pages 1374 - 1381, July 2014.

[47] Rumelhart, D et McClelland J. Parallel Distributed Processing- Explorations in Microstructure of Cognition. Vol 1: Foundations, vol 2: Psychological and Biological Models. Cambridge : MIT Press, 198

[48] Zadeh, L. Fuzzy sets. Information and Control, 8(3) :338 - 353.1965

[49] Zadeh, L Fay. "My Life and Travels with the Father of Fuzzy Logic". 1998, TSI Press, Albuquerque, NM.

[50] 3rd GPP, TS 36.211, V8.8.0, September 2009.

[51] IEEE publisher (ICTC), 2018 International Conference on. :860-865 Oct, 2018

\section{AUTHOR}

\section{Dr. Khaled H. Almotairi}

Khaled H. Almotairi received the B.Sc. degree from King Abdulaziz University, Jeddah, Saudi Arabia, in 2004 and the M.A.Sc. and Ph.D. degrees from the University of Waterloo, Waterloo, ON, Canada, in 2007 and 2012, respectively, all in electrical and computer engineering. He is currently an assistant professor in the Department of Computer Engineering, Umm Al-Qura University, Makkah, Saudi Arabia. From 2004 to 2005, he worked as a full-time Instructor in the College of Telecommunication and Electronics, Jeddah, Saudi Arabia. His research interests include channel allocation, performance analysis and evaluation, protocol design, transmission power control, ad

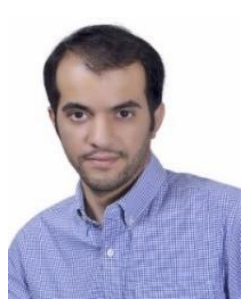
hoc networking. 